Supporting Information for

\title{
Cation-Disorder-Assisted Reversible Topotactic Phase Transition between Antifluorite and Rocksalt toward High-Capacity Lithium-Ion Batteries
}

Hiroaki Kobayashi* $†$ Takashi Tsukasaki, $\neq$ Yoshiyuki Ogasawara, $\neq$ Mitsuhiro Hibino, $\neq$

Tetsuichi Kudo, $\neq$ Noritaka Mizuno, $\neq$ Itaru Honma, $\uparrow$ and Kazuya Yamaguchi ${ }^{*}, \neq$

$\dagger$ Institute of Multidisciplinary Research for Advanced Materials, Tohoku University, 2-1-1 Katahira, Aoba-ku, Sendai, Miyagi, 980-8577, Japan.

\$Department of Applied Chemistry, School of Engineering, The University of Tokyo, 7-3-1 Hongo, Bunkyo-ku, Tokyo, 113-8656, Japan.

* E-mail: h.kobayashi@tohoku.ac.jp (H.K.), kyama@appchem.t.u-tokyo.ac.jp (K.Y.) ORCID: 0000-0001-6705-9515 (H.K.), 0000-0002-7661-4936 (K.Y.) 


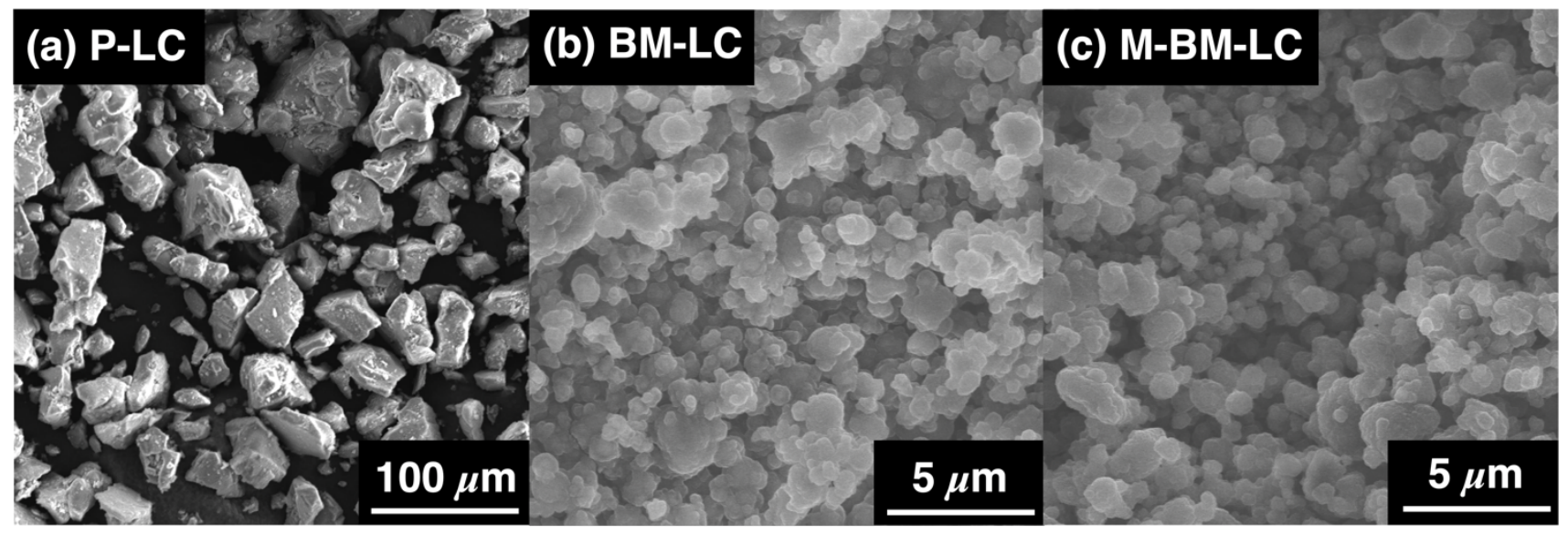

Figure S1. Low magnification SEM micrographs. (a) P-LC; (b) BM-LC; (c) M-BM-LC.
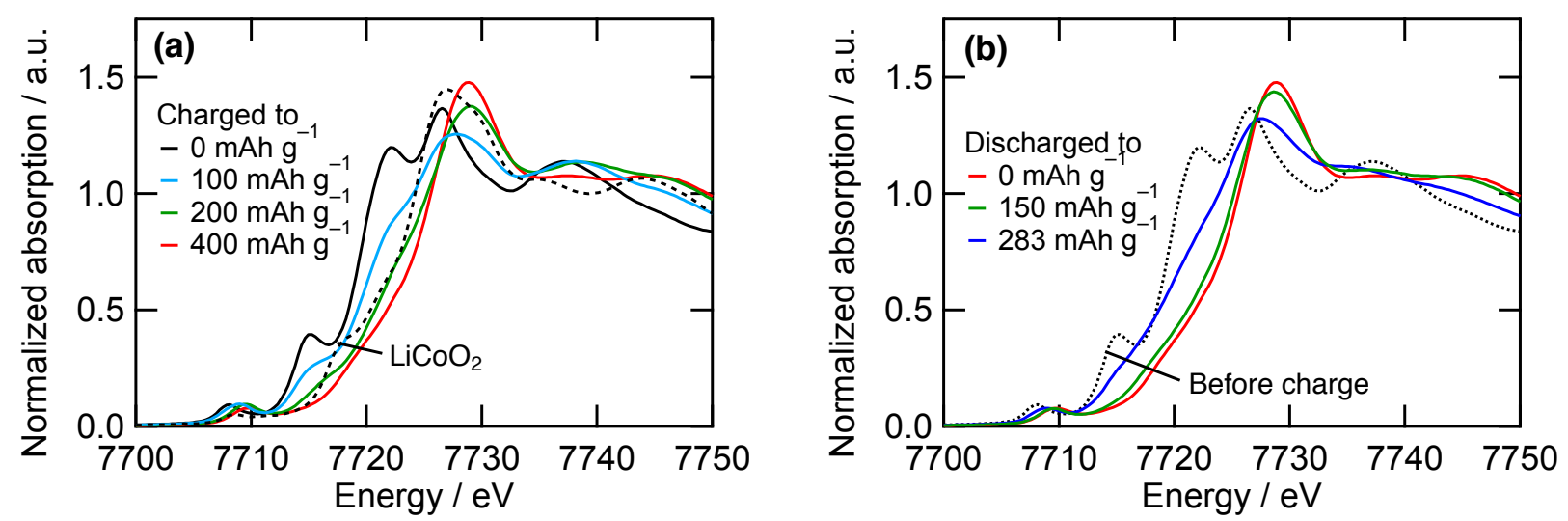

Figure S2. Co $K$-edge XANES spectra of P-LC. (a) During charge; (b) during dishcharge.

The edge energy at $400 \mathrm{mAh} \mathrm{g}^{-1}$-charge is higher than that of $\mathrm{LiCoO}_{2}$, indicating the oxidation from $\mathrm{Co}^{2+}$ to $\mathrm{Co}^{4+}$ occurs during charge. During discharge, there are little changes between the spectra at 0 and $150 \mathrm{mAh} \mathrm{g}^{-1}$-discharge. This suggests the reduction other than cationic occurs at higher voltage region. 


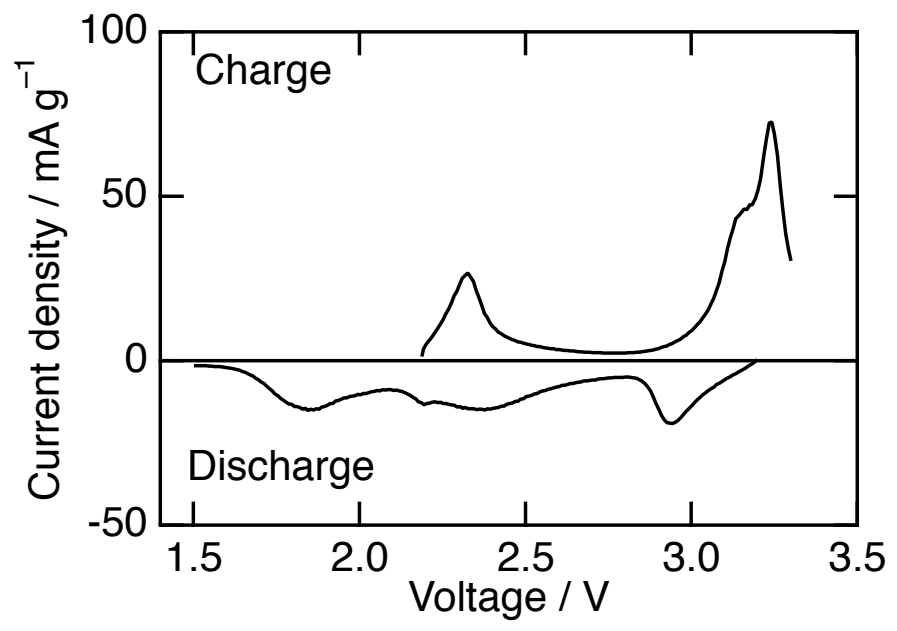

Figure S3. Linear sweep voltammogram of BM-LC. The cell was charged to $3.3 \mathrm{~V}$, and discharged to $1.5 \mathrm{~V}$ at $0.01 \mathrm{mV} / \mathrm{s}$. 
Table S1. Results of first and second coordination shell analysis in the Co K-edge EXAFS spectra of BM-LC during charge.

\begin{tabular}{llllllll}
\hline \multicolumn{5}{c}{ First coordination of Co-O } & \multicolumn{3}{c}{ Second coordination of Co-Co } \\
\hline $\begin{array}{l}\mathrm{Li}_{6-x} \mathrm{CoO}_{4} \\
\text { during } \\
\text { charge }\end{array}$ & $\begin{array}{l}\text { Coordination } \\
\text { number }\end{array}$ & $\begin{array}{l}\text { Distance } \\
(\AA)\end{array}$ & $\begin{array}{l}\text { Debye-Waller } \\
\text { factor }\left(\AA^{2}\right)\end{array}$ & $\begin{array}{l}\text { Coordination } \\
\text { number }\end{array}$ & $\begin{array}{l}\text { Distance } \\
(\AA)\end{array}$ & $\begin{array}{l}\text { Debye-Waller } \\
\text { factor }\left(\AA^{2}\right)\end{array}$ & R-factor $(\%)$ \\
\hline$x=0^{a}$ & $4.2(8)$ & $1.949(19)$ & $0.006(3)$ & - & - & - & 1.41 \\
$x=1$ & $4.2(6)$ & $1.840(14)$ & $0.0057(17)$ & $1.0(8)$ & $2.85(3)$ & $0.006(6)$ & 2.96 \\
$x=2$ & $5.0(6)$ & $1.863(10)$ & $0.0061(14)$ & $2.1(7)$ & $2.86(1)$ & $0.006(2)$ & 1.68 \\
$x=3$ & $5.3(4)$ & $1.871(7)$ & $0.0058(10)$ & $2.3(5)$ & $2.865(9)$ & $0.0060(17)$ & 0.81 \\
\hline
\end{tabular}

${ }^{a}$ First coordination shell analysis was applied with the $k$-range of the FT was 3-12 $\AA^{-1}$ with a Hanning window of $1 \AA^{-1}$, and the radial distance range of the inverse FT was $1-2 \AA$.

Table S2. Results of first and second coordination shell analysis in the Co $K$-edge EXAFS spectra of BM-LC during discharge.

\begin{tabular}{llllllll}
\hline & \multicolumn{5}{c}{ First coordination of Co-O } & \multicolumn{3}{c}{ Second coordination of Co-Co } \\
\hline $\begin{array}{l}\mathrm{Li}_{3+} \mathrm{CoO}_{4} \\
\text { during } \\
\text { discharge }\end{array}$ & $\begin{array}{l}\text { Coordination } \\
\text { number }\end{array}$ & $\begin{array}{l}\text { Distance } \\
(\AA)\end{array}$ & $\begin{array}{l}\text { Debye-Waller } \\
\text { factor }\left(\AA^{2}\right)\end{array}$ & $\begin{array}{l}\text { Coordination } \\
\text { number }\end{array}$ & $\begin{array}{l}\text { Distance } \\
(\AA)\end{array}$ & $\begin{array}{l}\text { Debye-Waller } \\
\text { factor }\left(\AA^{2}\right)\end{array}$ & R-factor $(\%)$ \\
\hline$x=0$ & $5.3(4)$ & $1.871(7)$ & $0.0058(10)$ & $2.3(5)$ & $2.865(9)$ & $0.0060(17)$ & 0.81 \\
$x=1$ & $5.1(5)$ & $1.870(7)$ & $0.0055(11)$ & $2.2(6)$ & $2.876(11)$ & $0.006(2)$ & 1.33 \\
$x=2$ & $5.0(6)$ & $1.878(9)$ & $0.0056(13)$ & $2.0(6)$ & $2.868(12)$ & $0.005(2)$ & 1.57 \\
$x=3$ & $4.7(5)$ & $1.926(11)$ & $0.0083(17)$ & $1.1(6)$ & $2.87(2)$ & $0.007(5)$ & 2.45 \\
\hline
\end{tabular}



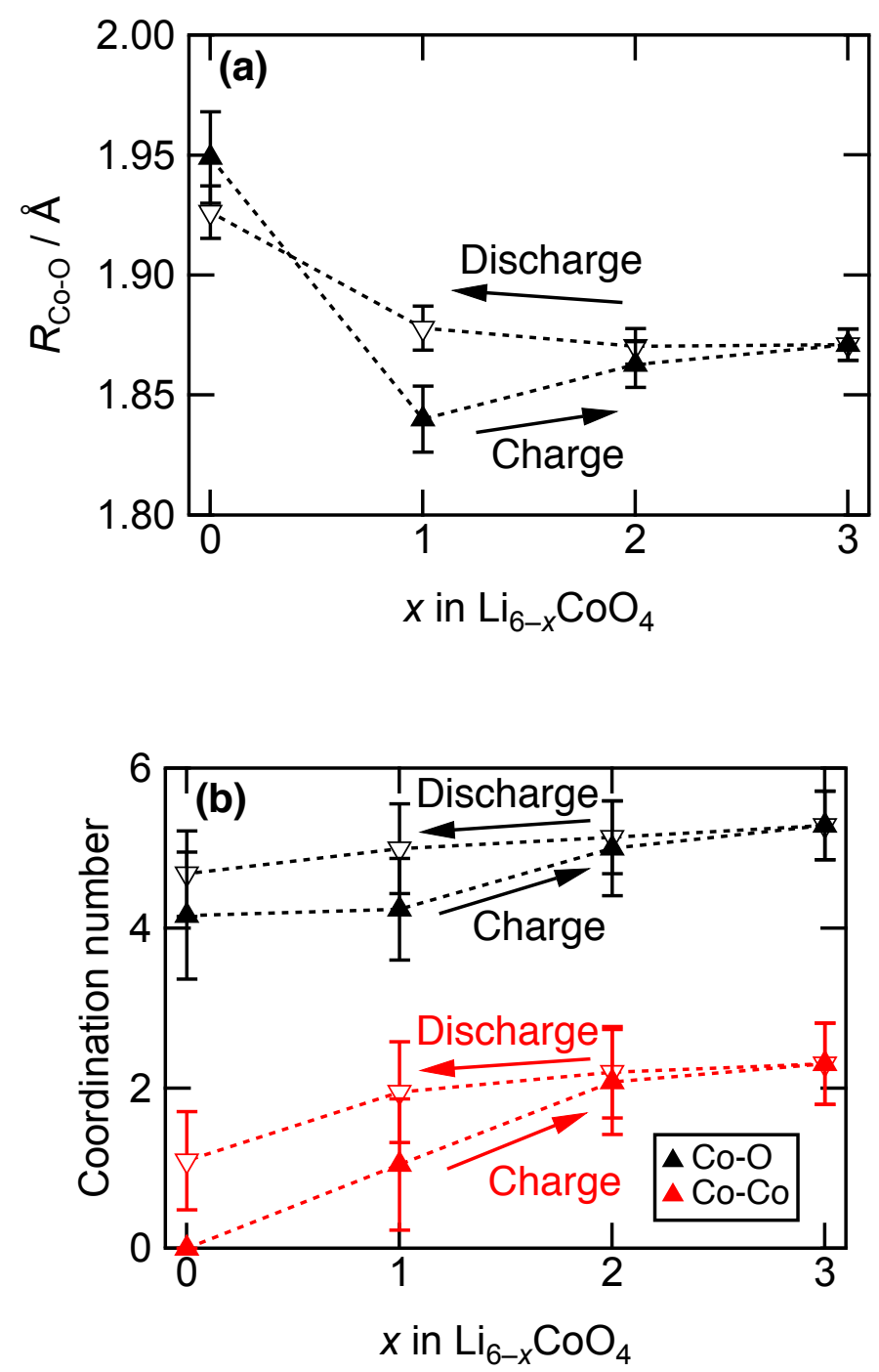

Figure S4. (a) Distance of Co-O and (b) coodination number of Co-O and Co-Co pairs estimated from the Co $K$-edge EXAFS analyses. 
Table S3. Results of Rietveld refinement for charged phase. ${ }^{a}$

Phase Space group Lattice parameters $(\AA)$ Atom Site Occupancy $(g)$

\begin{tabular}{llllll}
\hline $\mathrm{Li}_{2} \mathrm{CoO}_{3}$ & $F m \overline{3} m$ & $a=4.001(3)$ & $\mathrm{Li}$ & $4 b$ & $g=0.667(7)$ \\
& $\mathrm{Co}$ & $4 b$ & $g=0.333(7)$ \\
& $\mathrm{O}$ & $4 a$ & \\
& & &
\end{tabular}

${ }^{a} R_{\mathrm{wp}}=2.32 \%, R_{\mathrm{p}}=1.86 \%, R_{\mathrm{e}}=1.81 \%, S=1.28$.

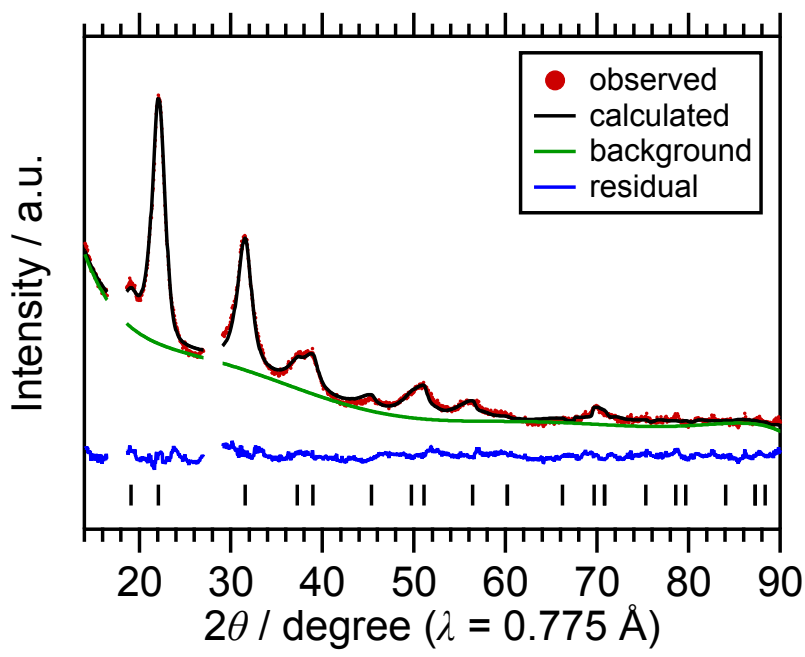

Figure S5. XRD pattern and fitting curve for charged electrode. 


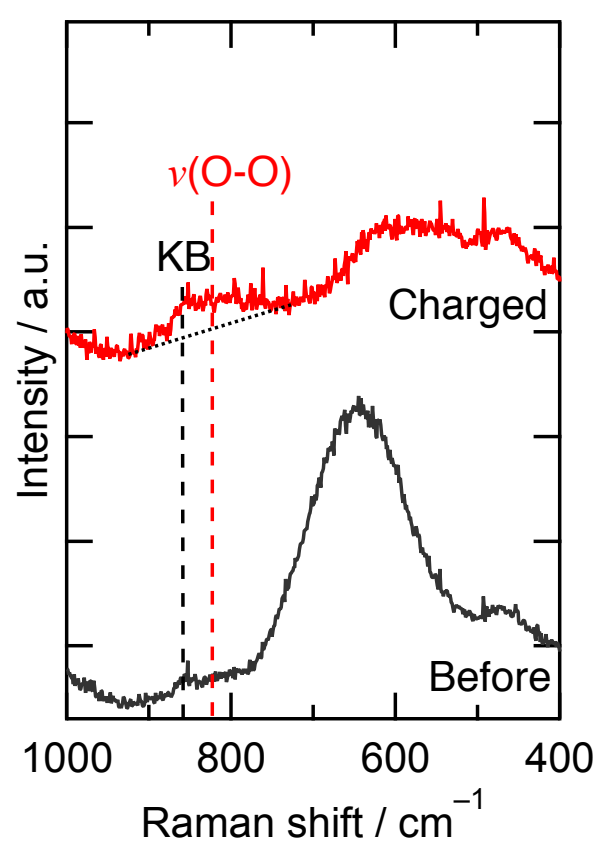

Figure S6. Raman spectra of BM-LC.

Table S4. Results of Rietveld refinement for M-BM-LC. ${ }^{a}$

\begin{tabular}{llllll}
\hline Phase & Space group & $\begin{array}{l}\text { Lattice } \\
\text { parameters }(\AA)\end{array}$ & Atom & Site & $\begin{array}{l}\text { Occupancy }(g) \text { and } \\
\text { atomic coordination }\end{array}$ \\
\hline$t$-LC & $P 4_{2} / n m c$ & $a=6.546(2)$ & Li1 & $8 f$ & $x=0.021(4)$ \\
& & Li2 & $4 d$ & $z=0.78(4)$ \\
& & Co & $2 a$ & \\
& & O & $8 g$ & $y=0.517(4)$ \\
& & & & $z=0.517(3)$
\end{tabular}

${ }^{a} R_{\mathrm{wp}}=2.63 \%, R_{\mathrm{p}}=1.90 \%, R_{\mathrm{e}}=1.16 \%, S=2.26$. 